\title{
Article \\ Gender-Specific Differences in Self-Care, Treatment-Related Symptoms, and Quality of Life in Hemodialysis Patients
}

\author{
Claudia Lerma ${ }^{1}{ }^{\mathbb{D}}$, Larissa I. Lima-Zapata ${ }^{1}$ (D), Jorge A. Amaya-Aguilar ${ }^{2}$, Itzel Leonardo-Cruz ${ }^{2}$, \\ Monica Lazo-Sánchez ${ }^{2}$, Luis A. Bermúdez ${ }^{3}$, Héctor Pérez-Grovas ${ }^{4}$, Abel Lerma ${ }^{5}$ (D) \\ and Julio César Cadena-Estrada ${ }^{2, *}$
}

check for updates

Citation: Lerma, C.; Lima-Zapata,

L.I.; Amaya-Aguilar, J.A.;

Leonardo-Cruz, I.; Lazo-Sánchez, M.; Bermúdez, L.A.; Pérez-Grovas, H.;

Lerma, A.; Cadena-Estrada, J.C. Gender-Specific Differences in

Self-Care, Treatment-Related

Symptoms, and Quality of Life in Hemodialysis Patients. Int. J. Environ. Res. Public Health 2021, 18, 13022. https://doi.org/10.3390/ ijerph182413022

Academic Editor: Paul B. Tchounwou

Received: 29 September 2021

Accepted: 8 December 2021

Published: 10 December 2021

Publisher's Note: MDPI stays neutral with regard to jurisdictional claims in published maps and institutional affiliations.

Copyright: (C) 2021 by the authors Licensee MDPI, Basel, Switzerland. This article is an open access article distributed under the terms and conditions of the Creative Commons Attribution (CC BY) license (https:// creativecommons.org/licenses/by/ $4.0 /)$
1 Department of Electromechanical Instrumentation, National Institute of Cardiology Ignacio Chávez, Mexico City 14080, Mexico; lermag@unam.mx (C.L.); larissalimazap94@gmail.com (L.I.L.-Z.)

2 Department of Nursing Research, National Institute of Cardiology Ignacio Chávez, Mexico City 14080, Mexico; skymanzeus@gmail.com (J.A.A.-A.); jitziunam@gmail.com (I.L.-C.); lzmonimar@gmail.com (M.L.-S.)

3 Centro Estatal de Hemodiálisis, Instituto Estatal de Cancerología, Colima 28000, Mexico; lbermudeza@hotmail.com

4 Department of Nephrology, National Institute of Cardiology Ignacio Chávez, Mexico City 14080, Mexico; hpgrovas@gmail.com

5 Institute of Health Sciences, Universidad Autónoma del Estado de Hidalgo, San Juan Tilcuautla, Pachuca 42160, Mexico; abel_lerma@uaeh.edu.mx

* Correspondence: jcadjul@gmail.com; Tel.: +52-55-5573-2911 (ext. 23300)

\begin{abstract}
Gender and sex differences affect women with kidney failure (KF) negatively at all stages of the disease. This study assessed gender differences in self-care, hemodialysis symptoms, and quality of life in a sample of 102 adult KF patients treated with hemodialysis, from two clinical centers in Mexico. Self-care agency, quality of life, and the symptoms related to hemodialysis were evaluated through questionnaires, and sociodemographic and laboratory variables were obtained from the clinical records. Compared to male patients, female patients reported similar self-care, lower quality of life subscales (symptoms, physical functioning, pain, and overall health), and higher prevalence and intensity of hemodialysis symptoms. There were gender differences regarding the correlation between self-care and quality of life, symptoms intensity, and symptoms prevalence. In conclusion, women with KF treated with hemodialysis perceived a higher impact of hemodialysis and reported a lower quality of life than men. Despite having a similar self-care agency, the self-care correlations with quality of life and hemodialysis symptoms appeared different between men and women treated with chronic hemodialysis. Such differences may be important in future nursing interventions to improve self-care and quality of life among KF patients.
\end{abstract}

Keywords: end-stage renal disease; gender role; hemodialysis symptoms; quality of life; self-care

\section{Introduction}

Chronic renal disease affects $10 \%$ of the global population. In Latin America, there is an increase in the prevalence of kidney failure (KF), which is associated with an accelerated incidence of patients with substitute renal treatment, including hemodialysis (HD) [1]. The quality of life (QoL) can decrease considerably in KF patients, which increases their mortality risk [2]. The aspects that are more affected in the QoL of KF patients are renal disease overload, physical function, social support, and overall health [3]. This points out the need for more planned and integral care from the team of health professionals for these patients.

The assessment of QoL includes several symptoms [4], but there is a questionnaire that achieves an integral evaluation of symptoms associated with HD [5]. Patients undergoing maintenance HD use to experience diverse physical symptoms (e.g., fatigue and lack of appetite) and emotional symptoms related to HD (e.g., depression and anxiety) [6]. 
Even though some symptoms can have an important severity [5], HD symptoms are usually underestimated by the health staff [7], and patients do not receive treatment for those symptoms [8]. The prevalence and severity of symptoms are associated with lower QoL [5]. Non-pharmacological strategies to improve QoL that consider patients symptoms are focused on some defined type of symptoms, either related to physical functioning (e.g., improving muscle strength through exercise) [9] or psycho-emotional (e.g., decreasing depressive and anxious symptoms through psychological interventions) [10]. Those interventions are effective for their therapeutic target [11] and involve some degree of active patient's participation, but they often do not consider the patient's perspective about their own ability to cope with multiple stressors inherent to their disease [12]. This could be crucial for their capability to successfully respond to the complex challenges of KF [12].

There are gender and sex differences that negatively affect women with KF from the initial diagnosis through access to a substitute renal treatment $[13,14]$, including renal transplant [15]. Some studies have shown lower QoL among women in HD [16]. Gender differences in self-care of renal patients have been studied scarcely. A study observed that women had similar self-care capacity than men, but they showed higher scores in self-efficacy [17]. It has also been reported that women in HD have better self-care efficacy of their arterio-venous fistula than men [18]. However, not all studies link the gender of HD patients with diverse self-efficacy aspects such as compliance to treatment [19].

Considering that the association between HD symptoms, self-care, and QoL has been studied scarcely, particularly from the perspective of gender, the aims of this exploratory work were: (i) to assess gender differences of self-care, HD symptoms, and QoL in KF patients treated with HD and (ii) to identify the associations between self-care, quality of life, and HD symptoms in these patients.

\section{Materials and Methods}

\subsection{Study Participants}

This exploratory cross-sectional study included 102 adult KF patients (55 men and 47 women) who were enrolled from two HD clinics located at Mexico City, during the first semester of 2017. All patients were adults, treated for at least a year with HD and had preserved cognitive function. This research complies with the principles described in the Declaration of Helsinki, and the study protocol was approved by the Research and Ethics Committees of our institution (protocol number 17-1015). The completed STROBE checklist is in the Supplementary Materials (File S1) [20]. The sampling method was nonprobabilistic. Since there are no previous studies about the correlation of the three main studied variables, we considered a sample size of 100 patients for the study, considering that a Pearson's correlation $\geq 0.35$ between any pair of the studied variables would be significant with an alpha error of 0.05 and a statistical power of $95 \%$.

\subsection{Study Protocol}

All eligible patients were invited during a personal interview; those interested in participating read the informed consent and were able to express their questions regarding the study. After written consent was obtained, each patient received a copy of the questionnaires and a detailed explanation on how to fill them in. Patients were able to fill the questionnaires in a well illuminated and ventilated room, in the quiet presence of a study researcher ready to answer any question. Clinical and laboratory results were obtained from the clinical records. Patients with incomplete data were eliminated from the study.

\subsection{Questionnaires}

Self-care was assessed with a Spanish language questionnaire of 43 items comprising three self-care agency dimensions development, operability, and adequacy [4]. The total self-care score was calculated in percentual units with the following equation: total selfcare $=($ self-care score -24$) \times 100) / 96$. The QoL was evaluated with the Kidney Disease 
Quality of Life Instrument (KdQoL) Version 1.3, which assesses QoL in KF patients with a renal replacement treatment [21]. The perceived HD symptoms were evaluated with the dialysis symptom index (DSI), which contains 30 items that assess the prevalence and intensity of physical and emotional symptoms associated with HD [5]. The Spanish language translation has reliability (Cronbach's alpha) of 0.889. A detailed description of the reliability analysis is in the Supplementary Materials (File S2). For this questionnaire, a correlation between HD symptoms and QoL in HD patients has been documented [6].

\subsection{Statistical Analysis}

Nominal variables are shown as absolute value and percentage and were compared regarding the CAC level by a chi-squared test. For ordinal variables, a KolmogorovSmirnov test was applied to verify normal distribution. Variables with normal distribution are shown as a mean \pm standard deviation and were compared between groups with a Student's $t$-test. Other variables are shown as median (percentile 25-percentile 75) and were compared between groups with a Mann-Whitney U test. The correlations between the selfcare and QoL questionnaires were calculated with the Spearman method. An analysis of variance was used to identify differences in the self-care score and the categorical variables compared by gender. The analysis was performed with the Statistical Package for Social Sciences (SPSS) version 21.0. A $p$-value $<0.05$ was considered statistically significant.

\section{Results}

Table 1 shows anthropomorphic characteristics, comorbidities, and laboratory results from the 102 study participants. Compared to men, women had lower serum hemoglobin, creatinine, blood urea nitrogen, uric acid, and sodium, as well as presented more cases with autoimmune diseases and allergies. Women also included more patients with immune disease as KF etiology, while in men, essential hypertension was a more frequent KF etiology. There were no significant differences among the other characteristics.

Table 1. Anthropometric characteristics, comorbidities, and laboratory results for 102 kidney failure (KF) patients treated with hemodialysis (HD) or hemodiafiltration. Data are shown as mean \pm standard deviation, median (percentile 25-percentile 75), or absolute frequency (percentage).

\begin{tabular}{cccc}
\hline Variables & $\begin{array}{c}\text { Women } \\
(\boldsymbol{N}=\mathbf{4 7})\end{array}$ & $\begin{array}{c}\text { Men } \\
(\boldsymbol{N}=\mathbf{5 5})\end{array}$ & $p$-Value \\
\hline Age (years) & $36.6 \pm 10.7$ & $39.3 \pm 14.1$ & 0.287 \\
Body mass index $\left(\mathrm{Kg} / \mathrm{m}^{2}\right)$ & $23.2 \pm 6.5$ & $23.9 \pm 6.6$ & 0.909 \\
Diagnosis time (months) & $5(3-12)$ & $6(3-12)$ & 0.886 \\
HD vintage (months) & $3(2-5)$ & $3(1-6)$ & 0.964 \\
Glucose $(\mathrm{mg} / \mathrm{dL})$ & $89(76-110)$ & $90(74-106)$ & 0.965 \\
Hemoglobin $(\mathrm{mg} / \mathrm{dL})$ & $8.75 \pm 1.67$ & $10.33 \pm 2.10$ & $<0.001$ \\
Creatinine $(\mathrm{mg} / \mathrm{dL})$ & $8.71 \pm 1.87$ & $11.87 \pm 3.42$ & 0.001 \\
Blood urea nitrogen $(\mathrm{mg} / \mathrm{dL})$ & $51.06 \pm 19.08$ & $61.83 \pm 21.6$ & 0.023 \\
Uric acid $(\mathrm{mg} / \mathrm{dL})$ & $5.94 \pm 1.14$ & $6.59 \pm 1.51$ & 0.056 \\
Albumin $(\mathrm{g} / \mathrm{dL})$ & $4.02 \pm 0.25$ & $4.12 \pm 0.28$ & 0.420 \\
Potassium $(\mathrm{mEq} / \mathrm{L})$ & $4.82 \pm 0.62$ & $4.70 \pm 0.72$ & 0.046 \\
Sodium $(\mathrm{mmol} / \mathrm{L})$ & $136.6 \pm 3.2$ & $137.8 \pm 2.7$ & 0.978 \\
Calcium $(\mathrm{mg} / \mathrm{dL})$ & $8.7 \pm 0.8$ & $8.7 \pm 1.0$ & 0.059 \\
Phosphorus (mg/dL) & $4.27 \pm 1.69$ & $5.02 \pm 2.09$ & \\
\hline KF etiology & & & 0.015 \\
Diabetes mellitus & $4(8.5 \%)$ & $5(9.1 \%)$ & \\
Essential hypertension & $2(4.3 \%)$ & $13(23.6 \%)$ & $2(3.6 \%)$ \\
Autoimmune disease & $7(14.9 \%)$ & $35(63.6 \%)$ & \\
Other & $34(72.3 \%)$ & &
\end{tabular}


Table 1. Cont.

\begin{tabular}{cccc}
\hline Variables & $\begin{array}{c}\text { Women } \\
(\boldsymbol{N}=47)\end{array}$ & $\begin{array}{c}\text { Men } \\
(\boldsymbol{N}=55)\end{array}$ & $p$-Value \\
\hline HD mode & & & \\
Hemodiafiltration & $21(44.7 \%)$ & $29(52.7 \%)$ & 0.418 \\
Hemodialysis & $26(55.3 \%)$ & $26(47.3 \%)$ & \\
\hline Vascular access & & & \\
Arterio-venous fistula & $21(46.7 \%)$ & $31(58.5 \%)$ & 0.437 \\
Tunneled catheter & $21(46.7 \%)$ & $18(34.0 \%)$ & \\
Non-tunneled catheter & $3(6.7 \%)$ & $4(7.5 \%)$ & 0.699 \\
Expecting renal transplant & $21(45.7 \%)$ & $23(41.8 \%)$ & 0.475 \\
Arterial hypertension & $31(70.5 \%)$ & $35(63.6 \%)$ & 0.929 \\
Diabetes mellitus & $8(17.0 \%)$ & $9(16.4 \%)$ & 0.027 \\
Cardiac disease & $7(14.9 \%)$ & $9(16.4 \%)$ & 0.909 \\
Autoimmune disease & $6(13.0 \%)$ & $1(1.8 \%)$ & 0.009 \\
Previous surgery & $1(2.2 \%)$ & $1(1.9 \%)$ & 0.874 \\
Food of drug allergies & $12(25.5 \%)$ & $6(10.9 \%)$ & 0.543 \\
Hepatitis B vaccine & $10(21.3 \%)$ & $11(20.0 \%)$ & 0.167 \\
Full vaccination scheme & $8(17.0 \%)$ & $12(21.8 \%)$ & 0.446 \\
Previous hospitalization & $13(27.7 \%)$ & $9(16.4 \%)$ & 0.080 \\
Blood transfusion & $17(36.2 \%)$ & $16(29.1 \%)$ & 0.133 \\
Smoking & $1(2.1 \%)$ & $6(10.9 \%)$ & \\
Alcohol use & $2(4.3 \%)$ & $7(12.7 \%)$ & \\
\hline
\end{tabular}

Table 2 shows that there were no significant differences between gender on demographic characteristics.

Table 2. Demographic characteristics of the study participants. Data are shown as mean \pm standard deviation, median (percentile 25-percentile 75), or absolute frequency (percentage).

\begin{tabular}{|c|c|c|c|}
\hline Variables & Women & Men & $p$-Value \\
\hline & $(N=47)$ & $(N=55)$ & \\
\hline Monthly income (pesos) & $4000(2000-5000)$ & $3200(2000-5000)$ & 0.878 \\
\hline $\begin{array}{l}\text { Commute time to clinic } \\
\text { (hours) }\end{array}$ & $1.00(0.75-0.62)$ & $1.00(0.66-0.74)$ & 0.742 \\
\hline Commute expenses (pesos) & $100(53-238)$ & $100(58-150)$ & 0.222 \\
\hline $\begin{array}{c}\text { Education } \\
\text { Illiterate } \\
\text { Primary/secondary school } \\
\text { High school/College }\end{array}$ & $\begin{array}{c}1(2.1 \%) \\
25(53.2 \%) \\
21(44.7 \%)\end{array}$ & $\begin{array}{c}1(1.8 \%) \\
30(54.5 \%) \\
24(43.6 \%)\end{array}$ & 0.986 \\
\hline $\begin{array}{c}\text { Marital status } \\
\text { Married or common-law } \\
\text { Single, widow(er), divorced }\end{array}$ & $\begin{array}{l}27(57.4 \%) \\
20(42.6 \%)\end{array}$ & $\begin{array}{l}27(49.1 \%) \\
28(50.9 \%)\end{array}$ & 0.399 \\
\hline $\begin{array}{c}\text { Work status } \\
\text { Unemployed } \\
\text { Remunerated work } \\
\text { Non-remunerated work }\end{array}$ & $\begin{array}{c}18(40.0 \%) \\
22(48.9 \%) \\
5(11.1 \%)\end{array}$ & $\begin{array}{c}14(26.4 \%) \\
33(62.3 \%) \\
6(11.3 \%)\end{array}$ & 0.341 \\
\hline $\begin{array}{c}\text { Family structure } \\
\text { Alone } \\
\text { Nuclear } \\
\text { Extensive }\end{array}$ & $\begin{array}{c}1(2.1 \%) \\
38(80.9 \%) \\
8(17.0 \%)\end{array}$ & $\begin{array}{c}2(3.6 \%) \\
43(78.2 \%) \\
10(18.2 \%)\end{array}$ & 0.888 \\
\hline
\end{tabular}


Table 2. Cont.

\begin{tabular}{cccc}
\hline Variables & Women & Men & $p$-Value \\
\hline Role in the family & $(\boldsymbol{N}=\mathbf{4 7 )}$ & $\mathbf{( N = 5 5 )}$ & \\
Father/mother & $22(47.8 \%)$ & $18(33.3 \%)$ & \\
Son/daughter & $14(30.4 \%)$ & $23(42.6 \%)$ & \\
Husband/wife & $9(19.6 \%)$ & $6(11.1 \%)$ & \\
Brother/sister & $1(2.2 \%)$ & $4(7.4 \%)$ & \\
Other & $0(0.0 \%)$ & $2(3.7 \%)$ & \\
House ownership & & & \\
Own & $23(48.9 \%)$ & $35(63.6 \%)$ & \\
Borrowed & $10(21.3 \%)$ & $10(18.2 \%)$ & 0.660 \\
Other & $14(29.8 \%)$ & $10(18.2 \%)$ & \\
\hline Health insurance system & & & \\
IMSS & $32(69.6 \%)$ & $37(67.3 \%)$ & 0.993 \\
ISSSTE & $1(2.2 \%)$ & $2(3.6 \%)$ & \\
Seguro popular & $5(10.9 \%)$ & $13(23 \%)$ & \\
None/other & $8(17.4 \%)$ & $14(25.5 \%)$ & \\
\hline Private health insurance & $12(25.5 \%)$ & & \\
\hline
\end{tabular}

IMSS: Instituto Mexicano del Seguro Social, ISSSTE: Instituto de Seguridad y Servicios Sociales de los Trabajadores del Estado.

The results of self-care, HD symptoms, and QoL are shown in Table 3. There were no differences by gender on the total self-care score. Compared to men, women had higher scores for prevalence and intensity of HD symptoms and lower QoL scores for the sub-scales of symptoms, physical functioning, pain, and overall health. There were no significant differences for the other QoL subscales or the total QoL scale.

Table 4 shows that self-care had a positive correlation in both genders with QoL (in the total scale and the subscales of physical functioning and fatigue/energy). Only in women, self-care had a positive correlation with QoL in the sub-scales of disease effect, disease load, cognitive functioning, social support, patient satisfaction, and emotional state. On the other side, only men showed a positive correlation between self-care and QoL in the sub-scale of sleep. There was no correlation between self-care and the other QoL subscales. The correlation analysis between HD symptoms and QoL showed that both genders had negative correlations with HD symptoms (prevalence and intensity) and QoL (total score and subscales of symptoms, effect, load, cognitive function, social interaction quality, pain, emotional state, and emotional role, social function, and fatigue/energy). Only in women, the prevalence and intensity of symptoms were negatively correlated with QoL in the physical role and overall health sub-scales. There was no correlation between HD symptoms and the other QoL sub-scales (work status, sexual functioning, positive interaction with the health staff, and patient satisfaction).

The associations between self-care, sociodemographic variables, and laboratory results were assessed with a Spearman correlation analysis (Table 5). There was no correlation between self-care and the evaluated variables, except for the transportation time from home to the HD clinic, which had a positive correlation with self-care in all patients but was not significant in the groups separated by gender. 
Table 3. Self-care, hemodialysis (HD) symptoms, and quality of life (QoL) of the study participants. Data are shown as mean \pm standard deviation or median (percentile 25-percentile 75 ).

\begin{tabular}{cccc}
\hline Variables & $\begin{array}{c}\text { Women } \\
(\mathbf{N}=\mathbf{4 7})\end{array}$ & $\begin{array}{c}\text { Men } \\
(\mathbf{N}=55)\end{array}$ & $p$-Value \\
\hline Self-care & $77.1 \pm 10.8$ & $77.5 \pm 9.9$ & 0.871 \\
HD symptoms & & & \\
Intensity (points) & $35.2 \pm 21.8$ & $22.2 \pm 16.5$ & 0.001 \\
Prevalence $\%$ ) & $12.7 \pm 6.5$ & $8.9 \pm 5.1$ & 0.001 \\
QoL & & & \\
Total score & $68.8 \pm 13.6$ & $73.4 \pm 11.1$ & 0.064 \\
Symptoms & $83.3(75.0-89.5)$ & $89.5(79.1-93.7)$ & 0.002 \\
Disease effect & $68.7(62.5-81.2)$ & $78.1(68.7-90.6)$ & 0.074 \\
Renal disease load & $50(25-69)$ & $50(31-75)$ & 0.688 \\
Work status & $50(50-100)$ & $50(0-100)$ & 0.721 \\
Cognitive function & $86.6(66.6-100.0)$ & $86.6(73.3-100.0)$ & 0.499 \\
Social interaction quality & $86.6(66.6-100.0)$ & $86.6(66.6-93.3)$ & 0.560 \\
Sexual function & $93.7(56.2-100.0)$ & $100.0(65.6-100.0)$ & 0.640 \\
Sleep & $56.6(50-63.3)$ & $60.0(53.3-63.3)$ & 0.114 \\
Social support & $83.3(66.6-100.0)$ & $83.3(66.6-100.0)$ & 0.543 \\
Positive interaction with & $75.0(62.5-100.0)$ & $87.5(75.0-100.0)$ & 0.105 \\
health staff & $83.3(50.0-100.0)$ & $83.3(66.6-100.0)$ & 0.608 \\
Patient's satisfaction & $70(40-80)$ & $75(60-90)$ & 0.033 \\
Physical functioning & $50(25-100)$ & $75(25-100)$ & 0.446 \\
Physical role & $68(55-90)$ & $88(68-100)$ & 0.039 \\
Pain & $50(45-65)$ & $65(50-75)$ & 0.041 \\
Overall health & $80(64-92)$ & $84(76-96)$ & 0.063 \\
Emotional status & $100(67-100)$ & $100(100-100)$ & 0.307 \\
Emotional role & $75(63-100)$ & $88(63-100)$ & 0.497 \\
Social function & $60(40-80)$ & $70(55-80)$ & 0.044 \\
Fatigue/energy & & &
\end{tabular}

Table 4. Spearman correlation coefficients for quality of life (QoL), self-care, and HD symptoms in 102 KF patients. Significant correlations $(p<0.05)$ are indicated with an asterisk $\left(^{*}\right)$.

\begin{tabular}{|c|c|c|c|c|c|c|}
\hline \multirow[b]{2}{*}{ Variables } & \multicolumn{2}{|c|}{ Self-Care } & \multicolumn{2}{|c|}{$\begin{array}{l}\text { HD Symptoms } \\
\text { Prevalence }\end{array}$} & \multicolumn{2}{|c|}{$\begin{array}{l}\text { HD Symptoms } \\
\text { Intensity }\end{array}$} \\
\hline & $\begin{array}{l}\text { Women } \\
(N=47)\end{array}$ & $\begin{array}{c}\text { Men } \\
(N=55)\end{array}$ & $\begin{array}{l}\text { Women } \\
(N=47)\end{array}$ & $\begin{array}{c}\text { Men } \\
(N=55)\end{array}$ & $\begin{array}{l}\text { Women } \\
(N=47)\end{array}$ & $\begin{array}{c}\text { Men } \\
(N=55)\end{array}$ \\
\hline Total score & $0.415^{*}$ & 0.293 * & $-0.539 *$ & $-0.512 *$ & $-0.580 *$ & $-0.513 *$ \\
\hline Symptoms & 0.167 & 0.040 & $-0.660 *$ & $-0.631 *$ & $-0.757 *$ & $-0.610 *$ \\
\hline Disease effect & $0.380 *$ & 0.137 & $-0.391 *$ & $-0.403 *$ & $-0.350 *$ & -0.409 * \\
\hline Renal disease load & $0.463 *$ & 0.228 & $-0.320 *$ & -0.294 * & $-0.317 *$ & -0.331 * \\
\hline Work status & 0.030 & 0.037 & -0.175 & -0.171 & -0.261 & -0.120 \\
\hline Cognitive function & 0.328 * & 0.002 & $-0.396 *$ & $-0.573 *$ & $-0.485^{*}$ & -0.556 * \\
\hline $\begin{array}{l}\text { Social interaction } \\
\text { quality }\end{array}$ & 0.151 & 0.216 & $-0.416^{*}$ & $-0.455^{*}$ & $-0.497^{*}$ & -0.453 * \\
\hline Sexual function & 0.297 & 0.091 & -0.112 & -0.165 & -0.240 & -0.237 \\
\hline Sleep & 0.155 & $0.375 *$ & $-0.334 *$ & $-0.363 *$ & $-0.446^{*}$ & -0.390 * \\
\hline Social support & $0.295 *$ & 0.158 & -0.261 & -0.173 & -0.246 & -0.114 \\
\hline $\begin{array}{l}\text { Positive interaction } \\
\text { with health staff }\end{array}$ & 0.284 & 0.132 & 0.133 & -0.082 & 0.159 & -0.011 \\
\hline Patient's satisfaction & $0.340 *$ & 0.021 & 0.062 & 0.003 & 0.113 & -0.001 \\
\hline Physical functioning & $0.297 *$ & $0.270 *$ & -0.249 & -0.233 & -0.196 & -0.204 \\
\hline Physical role & 0.163 & 0.158 & $-0.349 *$ & -0.104 & $-0.347 *$ & -0.119 \\
\hline Pain & 0.081 & 0.095 & $-0.546^{*}$ & $-0.479 *$ & $-0.564 *$ & $-0.485 *$ \\
\hline Overall health & 0.273 & 0.069 & $-0.295 *$ & -0.132 & $-0.333 *$ & -0.157 \\
\hline Emotional status & $0.297 *$ & 0.189 & $-0.603 *$ & $-0.468 *$ & $-0.669 *$ & $-0.469 *$ \\
\hline Emotional role & 0.048 & 0.158 & $-0.351 *$ & $-0.268 *$ & $-0.438 *$ & $-0.298 *$ \\
\hline Social function & 0.210 & 0.181 & $-0.493 *$ & $-0.377^{*}$ & $-0.488 *$ & $-0.392 *$ \\
\hline Fatigue/energy & $0.300 *$ & $0.272 *$ & $-0.552 *$ & $-0.450 *$ & $-0.575^{*}$ & -0.419 * \\
\hline
\end{tabular}


Table 5. Spearman correlation coefficients between self-care and ordinal variables. Significant correlation $(p<0.05)$ is indicated with an asterisk $\left({ }^{*}\right)$.

\begin{tabular}{cccc}
\hline Variables & $\begin{array}{c}\text { All Patients } \\
(\mathbf{N}=\mathbf{1 0 2})\end{array}$ & $\begin{array}{c}\text { Women } \\
(\mathbf{N}=\mathbf{4 7})\end{array}$ & $\begin{array}{c}\text { Men } \\
(\mathbf{N}=\mathbf{5 5})\end{array}$ \\
\hline Age (years) & 0.058 & 0.009 & 0.093 \\
Body mass index $\left(\mathrm{Kg} / \mathrm{m}^{2}\right)$ & -0.190 & -0.113 & -0.259 \\
Diagnosis time (months) & 0.051 & 0.104 & -0.023 \\
HD vintage (months) & -0.078 & -0.086 & -0.105 \\
Hemoglobin $(\mathrm{mg} / \mathrm{dL})$ & -0.049 & 0.014 & -0.061 \\
Glucose $(\mathrm{mg} / \mathrm{dL})$ & -0.185 & -0.257 & -0.136 \\
Creatinine $(\mathrm{mg} / \mathrm{dL})$ & -0.056 & -0.227 & 0.032 \\
Blood urea nitrogen (mg/dL) & -0.001 & -0.115 & 0.123 \\
Uric acid (mg/dL) & -0.121 & -0.179 & -0.062 \\
Albumin (g/dL) & 0.170 & 0.146 & 0.194 \\
Potassium (mEq/L) & 0.028 & -0.139 & 0.163 \\
Sodium (mmol/L) & 0.043 & 0.119 & 0.024 \\
Calcium (mg/dL) & -0.093 & -0.072 & -0.122 \\
Phosphorous (mg/dL) & -0.081 & -0.070 & -0.108 \\
Monthly income & -0.009 & 0.017 & -0.044 \\
Commute time to clinic & $0.213 *$ & 0.206 & 0.220 \\
(hours) & 0.094 & 0.167 & 0.027 \\
Commute expenses (pesos) & -0.050 & -0.175 & 0.019 \\
Prevalence HD symptoms & -0.018 & -0.081 & 0.003 \\
Intensity HD symptoms & & & \\
\hline & & &
\end{tabular}

Table 6 shows that for nominal variables, only in the group of women, diabetic patients had a lower self-care score than non-diabetic women, and patients treated with hemodiafiltration had a higher self-care score than those treated with HD. There were no differences by gender for the other nominal variables.

Table 6. Self-care score compared by gender for the studied nominal variables.

\begin{tabular}{|c|c|c|}
\hline Variable & Women & Men \\
\hline & $(N=47)$ & $(N=55)$ \\
\hline \multicolumn{3}{|c|}{ Diabetes Mellitus } \\
\hline Yes & $69.9 \pm 17.5$ & $77.7 \pm 8.1$ \\
\hline No & $78.7 \pm 8.6^{\mathrm{a}}$ & $77.5 \pm 10.4$ \\
\hline \multicolumn{3}{|c|}{ Arterial hypertension } \\
\hline Yes & $75.4 \pm 11.8$ & $77.4 \pm 10.2$ \\
\hline No & $80.6 \pm 7.9$ & $77.6 \pm 9.7$ \\
\hline \multicolumn{3}{|c|}{ Cardiac disease } \\
\hline Yes & $72 \pm 11.7$ & $75.9 \pm 10.4$ \\
\hline No & $78 \pm 10.62$ & $77.8 \pm 9.9$ \\
\hline \multicolumn{3}{|l|}{ Allergy } \\
\hline Yes & $78.1 \pm 13.8$ & $74.3 \pm 3.5$ \\
\hline No & $76.8 \pm 9.8$ & $77.8 \pm 10.4$ \\
\hline \multicolumn{3}{|c|}{ Previous hospitalization } \\
\hline Yes & $76.1 \pm 12.0$ & $80.7 \pm 9.6$ \\
\hline No & $77.5 \pm 10.5$ & $76.8 \pm 10.0$ \\
\hline \multicolumn{3}{|c|}{ Previous transfusion } \\
\hline Yes & $76.4 \pm 11.8$ & $80.3 \pm 7.8$ \\
\hline No & $77.5 \pm 10.4$ & $76.3 \pm 10.5$ \\
\hline \multicolumn{3}{|c|}{ Alcohol drinking } \\
\hline Yes & $75 \pm 8.2$ & $80 \pm 7.6$ \\
\hline No & $77.2 \pm 11$ & $77.1 \pm 10.2$ \\
\hline
\end{tabular}


Table 6. Cont.

\begin{tabular}{|c|c|c|}
\hline Variable & Women & Men \\
\hline & $(N=47)$ & $(N=55)$ \\
\hline \multicolumn{3}{|l|}{ Education } \\
\hline $\begin{array}{l}\text { Illiterate, primary or } \\
\text { secondary }\end{array}$ & $75.2 \pm 13$ & $77.1 \pm 9.7$ \\
\hline High school or college & $79.5 \pm 6.9$ & $77.9 \pm 10.4$ \\
\hline \multicolumn{3}{|l|}{ Marital status } \\
\hline $\begin{array}{c}\text { Married/common-law } \\
\text { partner }\end{array}$ & $77 \pm 10.4$ & $78 \pm 10.6$ \\
\hline Single/widow(er)/divorced & $77.3 \pm 11.6$ & $77 \pm 9.4$ \\
\hline \multicolumn{3}{|l|}{ Work status } \\
\hline Remunerated work & $73.1 \pm 10.5$ & $78.4 \pm 10.1$ \\
\hline $\begin{array}{l}\text { Unemployed/Non- } \\
\text { remunerated }\end{array}$ & $75.5 \pm 11.0$ & $76 \pm 10.1$ \\
\hline \multicolumn{3}{|l|}{ House ownership } \\
\hline Own & $76.8 \pm 14.1$ & $76.1 \pm 11.1$ \\
\hline Borrowed & $79.4 \pm 6.8$ & $79.4 \pm 81.6$ \\
\hline Other & $76.1 \pm 6.5$ & $76.1 \pm 8$ \\
\hline \multicolumn{3}{|l|}{ Health insurance system } \\
\hline Government agency & $77.4 \pm 10.06$ & $76.7 \pm 10.1$ \\
\hline None/other & $74.1 \pm 14.3$ & $79.8 \pm 9.2$ \\
\hline \multicolumn{3}{|l|}{ Private health insurance } \\
\hline Yes & $77.2 \pm 9.8$ & $79.1 \pm 10.1$ \\
\hline No & $77.1 \pm 11.3$ & $76.93 \pm 9.9$ \\
\hline \multicolumn{3}{|l|}{ Hemodialysis mode } \\
\hline Hemodiafiltration & $80.6 \pm 6.9$ & $78.5 \pm 7.8$ \\
\hline Hemodialysis & $74.3 \pm 12.6^{b}$ & $76.3 \pm 11.9$ \\
\hline \multicolumn{3}{|l|}{ Vascular access } \\
\hline Arterio-venous fistula & $79.4 \pm 9.6$ & $78.1 \pm 7.5$ \\
\hline Tunneled catheter & $74.7 \pm 12.1$ & $73.6 \pm 12.4$ \\
\hline Non-tunneled catheter & $81.1 \pm 13.0$ & $86 \pm 10.5$ \\
\hline
\end{tabular}

\section{Discussion}

Our results show that in most patients (both men and women) a higher self-care was associated with a higher QoL on physical functioning and fatigue/energy. Similar results from Trask et al. [22] showed that with higher self-care, the QoL was better and that an increase in HD knowledge by the patients and self-care promotion in their treatment decreased their one-year mortality. A previous report showed that patients in chronic HD consider physical activity as an important self-care factor to maintain their health, but at the same time, they consider that an important barrier to practicing physical activity is the fatigue attributed both to the disease and to the HD treatment [23]. The present study shows that, although there was a significant association between physician functioning and self-care for both genders (Table 4), female patients reported a greater impact in both physical functioning and fatigue/energy compared to male patients (Table 3).

Bettoni et al. [24] reported a positive relation of moderate magnitude between self-care and several QoL subscales (symptoms/problems, cognitive functioning, physical function, emotional well-being, energy/fatigue, depression, and anxiety). These results are similar to our findings, which reinforces the need for health professionals, including nurses, to provide interventions aimed at both the patient and the primary caregiver regarding their knowledge of HD, HD functioning, medication intake difficulties, allowed and prohibited foods, fluids intake, venous access care, prevention of HD symptoms, leisure, interpersonal 
relationships, HD complications, anti-coagulation treatments, the practice of emotional activities, and association with social groups.

We observed that the disease load, emotional state, and social support were the main factors related to self-care. Among many factors, these correlations could be explained not only by KF itself and HD but also by patients' perception of the arterio-venous fistula. According to Silva et al. [25], venous access through a fistula leaves marks that alter the body's aesthetics, making the body imperfect. These changes cause low self-esteem and attract the look of others. This gives discomfort to HD patients, who try to hide their fistula and at the same time recognize the fistula as essential for their own life. From this perception arises fear in the patient, as a catalyst for self-care. We found not gender differences in the vascular access type in the sample of the present study. However, previous studies report that gender female patients starting chronic HD are less likely to have access to an arteriovenous fistula than men, and females with an arteriovenous fistula have a higher rate of 1-year survival than those who do not have this vascular access [26]. Therefore, understanding the potential effect of this gender disparity on self-care and quality of life warrants further studies.

Previous works have documented the influence of variables that promote self-care behavior during HD, including knowledge [27], attitudes, ability, previous self-efficacy, motivation, higher scholarly, having a stable job, and a smaller number of HD sessions [28]. The nursing staff must consider these factors to develop nursing consultation so to improve therapeutic adherence and self-care, mainly focused on diet and medication and linked to a long involvement in the treatment and a more frequent contact with the primary caregiver [29].

In our study, the patients with higher self-care perception were treated with HD or hemodiafiltration and required a longer commute from home to the HD unit. Probably, the perceived self-care is linked to their ability to organize and perform the actions needed to achieve a certain degree of performance (e.g., to be able to arrive on time despite a long commute), and therefore these beliefs influence their goal-oriented behavior, such as the choice of a task, the persistence, the effort, and the acquisition of abilities [30].

Previous work has reported that women treated with HD had higher self-care than men [18], while others have found similar self-care between men and women but a lower self-efficacy in female patients [17]. In most cases, the onset of a terminal disease such as KF requires prolonged treatment, and the main caregiver is a member of the family [31]. Lee et al. [32] showed that HD-treated patients with family caregivers reported higher self-care scores than those without a family caregiver and found a significant correlation between self-care and social support. When we evaluated such correlation separately by gender, the correlation was significant only for female patients (Table 4). Further, it is known that if the patient is a man, the caregiver is often a woman (his wife, daughter, or mother), but if the patient is a woman, the caregiver usually is her daughter or her mother [31]. Moreover, in Latin American countries such as Mexico, where culture influences the housekeeping process, most women who become ill continue performing their roles as mother, daughter, or wife, prioritizing the care of others before their own care [33]. Therefore, it is necessary that health professionals, especially the nursing staff, recognize their educational role and develop strategies to teach renal patients about self-care, converting them into protagonists of the therapeutic process, including their family. This will contribute to the implementation of safe practices regarding the compliance to food intake and fluid intake restrictions, the balance between rest and physical activity, and the self-care of the vascular access and the fistula (e.g., during blood pressure measurements, drug administration, and when carrying weight with the arm).

One study limitation is the relatively small sample of participants, who were consecutively enrolled in two HD centers. Further studies with larger randomly selected samples from multiple HD centers are needed to increase the external validity of our findings and to confirm the absence of significance in several results of the present study. Future studies 
could use our preliminary findings to estimate the sample size needed to warrant a given statistical power.

\section{Conclusions}

Women with KF treated with HD perceived a higher impact of HD and reported a lower QoL than men. Despite having a similar self-care agency, the self-care correlations with QoL and HD symptoms resulted to be different between men and women treated with chronic HD. Such differences may be important in future nursing interventions to improve self-care and QoL among KF patients.

Supplementary Materials: The following are available online at https:/ /www.mdpi.com/article/10 .3390/ijerph182413022/s1, File S1: Completed STROBE checklist (S1); File S2: Results of the reliability analysis of the Spanish version of the Dialysis Symptoms Index (DSI).

Author Contributions: Conceptualization, C.L., J.A.A.-A. and J.C.C.-E.; methodology, C.L., J.A.A.-A. and J.C.C.-E.; software, C.L.; validation, H.P.-G. and L.A.B.; formal analysis, C.L., A.L. and L.I.L.-Z; investigation, C.L., J.A.A.-A., I.L.-C., M.L.-S. and J.C.C.-E.; resources, C.L., H.P.-G., L.A.B. and J.C.C.-E.; data curation, I.L.-C. and M.L.-S.; writing-original draft preparation, C.L., J.A.A.-A. and J.C.C.-E.; writing-review and editing, C.L., L.I.L.-Z., I.L.-C., M.L.-S., L.A.B., H.P.-G., J.A.A.-A., A.L. and J.C.C.-E.; visualization, L.I.L.-Z. and C.L.; supervision, C.L. and J.C.C.-E.; project administration, C.L. and J.C.C.-E.; funding acquisition, J.C.C.-E. All authors have read and agreed to the published version of the manuscript.

Funding: This research received no external funding. The Instituto Nacional de Cardiología Ignacio Chávez supported the open access funding.

Institutional Review Board Statement: The study was conducted according to the guidelines of the Declaration of Helsinki and approved by the Research and Ethics Committees of the National Institute of Cardiology Ignacio Chávez (protocol number 17-1015).

Informed Consent Statement: Each participating patient was informed about the characteristics of the study and signed a written informed consent.

Data Availability Statement: The data presented in this study are available on request from the corresponding author.

Acknowledgments: The authors thank the valuable collaboration of the health staff and patients at the participant HD clinics. This study was not supported by external funds.

Conflicts of Interest: The authors declare no conflict of interest.

\section{References}

1. Rosa-Diez, G.; Gonzalez-Bedat, M.; Ferreiro, A.; Garcia, G.G.; Fernandez-Cean, J.; Douthat, W. Burden of end-stage renal disease (ESRD) in Latin America. Clin. Nephrol. 2016, 86, 29-33. [CrossRef]

2. Pei, M.; Aguiar, R.; Pagels, A.A.; Heimbürger, O.; Stenvinkel, P.; Bárány, P.; Medin, C.; Jacobson, S.H.; Hylander, B.; Lindholm, B.; et al. Health-related quality of life as predictor of mortality in end-stage renal disease patients: An observational study. BMC Nephrol. 2019, 20, 1-10. [CrossRef]

3. Ageborg, M.; Allenius, B.L. Quality of life, self-care ability, and sense of coherence in hemodialysis patients: A comparative study. Hemodial Int. 2005, 9, 8-14. [CrossRef] [PubMed]

4. Gallegos, C.; Gallegos, E. Validez y confiabilidades de la versión en español de la escala: Valoración de las Capacidades de Autocuidado. Revista Enfermería 1998, 27, 38-47.

5. Weisbord, S.D.; Fried, L.F.; Arnold, R.M.; Fine, M.J.; Levenson, D.J.; Peterson, R.A.; Switzer, G.E. Prevalence, Severity, and Importance of Physical and Emotional Symptoms in Chronic Hemodialysis Patients. J. Am. Soc. Nephrol. 2005, 16, $2487-2494$. [CrossRef]

6. Abdel-Kader, K.; Unruh, M.L. Symptom Burden, Depression, and Quality of Life in Chronic and End-Stage Kidney Disease. Clin. J. Am. Soc. Nephrol. 2009, 4, 1057-1064. [CrossRef]

7. Weisbord, S.D.; Fried, L.F.; Mor, M.K.; Resnick, A.L.; Unruh, M.L.; Palevsky, P.M.; Levenson, D.J;; Cooksey, S.H.; Fine, M.J.; Kimmel, P.L.; et al. Renal Provider Recognition of Symptoms in Patients on Maintenance Hemodialysis. Clin. J. Am. Soc. Nephrol. 2007, 2, 960-967. [CrossRef] [PubMed]

8. Claxton, R.N.; Blackhall, L.; Weisbord, S.D.; Holley, J.L. Undertreatment of Symptoms in Patients on Maintenance Hemodialysis. J. Pain Symptom Manag. 2010, 39, 211-218. [CrossRef] 
9. Tarca, B.; Wycherley, T.; Bennett, P.; Meade, A.; Ferrar, K. Modifiable Physical Factors Associated with Physical Functioning for Patients Receiving Dialysis: A Systematic Review. J. Phys. Act. Heal. 2020, 17, 475-489. [CrossRef] [PubMed]

10. Lerma, A.; Perez-Grovas, H.; Bermudez, L.; Peralta, M.L.; Robles-García, R.; Lerma, C. Brief cognitive behavioural intervention for depression and anxiety symptoms improves quality of life in chronic haemodialysis patients. Psychol. Psychother. Theory Res. Pr. 2017, 90, 105-123. [CrossRef]

11. Wen, X.; Wang, Y. Nonpharmacological Interventions for Depressive Symptoms in End-Stage Renal Disease: A Systematic Review. West J. Nurs. Res. 2019, 965, 1-25. [CrossRef]

12. Cukor, D.; Cohen, S.D.; Peterson, R.A.; Kimmel, P.L. Psychosocial Aspects of Chronic Disease: ESRD as a Paradigmatic Illness. J. Am. Soc. Nephrol. 2007, 18, 3042-3055. [CrossRef]

13. Cobo, G.; Hecking, M.; Port, F.K.; Exner, I.; Lindholm, B.; Stenvinkel, P.; Carrero, J.J. Sex and gender differences in chronic kidney disease: Progression to end-stage renal disease and haemodialysis. Clin. Sci. 2016, 130, 1147-1163. [CrossRef] [PubMed]

14. Piccoli, G.B.; Alrukhaimi, M.; Liu, Z.-H.; Zakharova, E.; Levin, A. What we do and do not know about women and kidney diseases; questions unanswered and answers unquestioned: Reflection on World Kidney Day and International Woman's Day. Nefrología 2018, 38, 114-124. [CrossRef] [PubMed]

15. Melk, A.; Schmidt, B.M.W.; Geyer, S.; Epping, J. Sex disparities in dialysis initiation, access to waitlist, transplantation and transplant outcome in German patients with renal disease-A population based analysis. PLoS ONE 2020, 15, e0241556. [CrossRef]

16. Özdemir, A.A.; Sayın, C.B. Quality of life through gender role perspective in candidate renal transplant recipients: A report from başkent university using the short form 36 health survey. Exp. Clin. Transpl. 2018, 16, 28-34. [CrossRef]

17. Bağ, E.; Mollaoglu, M. The evaluation of self-care and self-efficacy in patients undergoing hemodialysis. J. Eval. Clin. Pr. 2009, 16, 605-610. [CrossRef] [PubMed]

18. Sousa, C.N.; Marujo, P.; Teles, P.; Lira, M.N.; Novais, M.E.L.M. Self-Care on Hemodialysis: Behaviors with the Arteriovenous Fistula. Ther. Apher. Dial. 2017, 21, 195-199. [CrossRef] [PubMed]

19. Loghman-Adham, M. Medication noncompliance in patients with chronic disease: Issues in dialysis and renal transplantation. Am. J. Manag. Care. 2015, 9, 155-171.

20. Von Elm, E.; Altman, D.G.; Egger, M.; Pocock, S.J.; Gøtzsche, P.C.; Vandenbroucke, J.P. The Strengthening the Reporting of Observational Studies in Epidemiology (STROBE) statement: Guidelines for reporting observational studies. J. Clin. Epidemiol. 2008, 61, 344-349. [CrossRef]

21. Franco, L.C.; Zatta, L.T.; Vasconcelos, P.; Barbosa, M.A.; Dos Santos, J.S.; Rosa, L.D.O. Evaluación de la calidad de vida de pacientes de insuficiencia renal crónica en diálisis renal. Enfermería Global 2011, 10, 158-164. [CrossRef]

22. Trask, M.-A.; Marchuk, S. The Evaluation of an Orientation Program of Self-Care Abilities for Patients on Hemodialysis. Nephrol. Nurs. J. 2016, 43, 501-534. Available online: https://www.ncbi.nlm.nih.gov/pubmed/30550079 (accessed on 18 March 2021).

23. Santana, M.B.A.; da Silva, D.M.G.V.; Echevarría-Guanilo, M.E.; Lopes, S.G.R.; Romanoski, P.J.; Böell, J.E.W. Self-care in individuals with chronic kidney disease on hemodialysis. Revista Gaúcha de Enfermagem 2020, 41, e20190220. [CrossRef]

24. Bettoni, L.C.; Ottaviani, A.C. Associação Entre o Autocuidado e a Qualidade de vida de Pacientes com Doença Renal Crônica. Rev. Eletrônica Enferm. 2017, 29, 19. Available online: https://revistas.ufg.br/fen/article/view/27442 (accessed on 18 March 2021). [CrossRef]

25. Silva, D.M.; Silva, R.M.C.R.A. The body marked by the arteriovenous fistula: A phenomenological point of view. Rev. Bras. Enferm. 2018, 71, 2869-2875. Available online: http:/ / www.scielo.br/scielo.php?script=sci_arttext\&pid=S0034-7167201800060286 9\&lng=en\&tlng=en (accessed on 18 March 2021). [CrossRef] [PubMed]

26. Angelici, L.; Marino, C.; Umbro, I.; Bossola, M.; Calandrini, E.; Tazza, L.; Agabiti, N.; Davoli, M.; Regional Registry Dialysis and Transplant Lazio Region. Gender Disparities in Vascular Access and One-Year Mortality among Incident Hemodialysis Patients: An Epidemiological Study in Lazio Region, Italy. J. Clin. Med. 2021, 10, 5116. [CrossRef]

27. Wu, S.V.; Hsieh, N.; Lin, L.; Tsai, J. Prediction of self-care behaviour on the basis of knowledge about chronic kidney disease using self-efficacy as a mediator. J. Clin. Nurs. 2016, 25, 2609-2618. [CrossRef]

28. Yang, M.; Zhao, H.; Ding, X.; Zhu, G.; Yang, Z.; Ding, L.; Yang, X.; Zhao, Y.; Chen, L.; Yang, R.; et al. Self-Care Behavior of Hemodialysis Patients with Arteriovenous Fistula in China: A Multicenter, Cross-Sectional Study. Ther. Apher. Dial. 2019, 23, 167-172. [CrossRef] [PubMed]

29. Ramírez, M.D.O.; Rodríguez, I.C.; Ramírez, D.O.; Pérez, A.M.G.; Hita, S.G.; Marcos, S.G. Consulta de enfermería y adherencia terapéutica del paciente en hemodiálisis. Enfermería Nefrológica 2017, 20, 132-138. [CrossRef]

30. Jayanti, A.; Foden, P. Illness beliefs in end stage renal disease and associations with self-care modality choice. PLoS ONE 2016, 11, 1-17. [CrossRef]

31. Belasco, A.G.; Sesso, R. Burden and quality of life of caregivers for hemodialysis patients. Am. J. Kidney Dis. 2002, 39, 805-812. [CrossRef] [PubMed]

32. Lee, J.; Noh, D. Factors associated with self-care among patients receiving hemodialysis: A cross-sectional observational study. Res. Theory Nurs. Pract. 2021. ahead of print. [CrossRef] [PubMed]

33. DiGirolamo, A.M.; De Snyder, N.S. Women as primary caregivers in Mexico: Challenges to well-being. Salud Pública México 2008, 50, 516-522. [CrossRef] [PubMed] 